\title{
Zur Theorie der Raumgeraden und der linearen Complexe.
}

Von v. Dracil in Marhurg.

Die aus den jedesmaligen Constanten der Gleichungen

$$
\begin{aligned}
& u_{0} x_{0}+u_{1} x_{1}+u_{2} x_{2}+u_{3} x_{3}=0 \\
& v_{0} y_{0}+v_{1} y_{1}+v_{2} y_{2}+v_{3} y_{3}=0
\end{aligned}
$$

(welche, mag man die rechtwinkligen Verhältnisscoordinateu der Punkte $x$ und $y$, oder die der Ebenen $u$ und $v$ variabel annehmeñ, eine Gerade im Raume darstellen) gebildeten Ausdrücke:

respective

$$
\begin{aligned}
& u_{0} v_{1}-u_{1} v_{0}, u_{1} v_{2}-u_{2} v_{1}, u_{2} v_{0}-u_{0} v_{2}, \\
& u_{0} v_{3}-u_{3} v_{0}, u_{1} v_{3}-u_{3} v_{1}, u_{2} v_{3}-u_{3} v_{2}
\end{aligned}
$$

$$
\begin{aligned}
& x_{0} y_{1}-x_{1} y_{0}, x_{1} y_{2}-x_{2} y_{1}, x_{2} y_{0}-x_{0} y_{2}, \\
& x_{0} y_{3}-x_{3} y_{0}, x_{1} y_{3}-x_{3} y_{1}, x_{2} y_{3}-x_{3} y_{2},
\end{aligned}
$$

die man abkürzend bezeichnet hat mit

und

$$
q_{i k} \equiv u_{i} v_{k}-u_{k} v_{i} \text {, wobei also } q_{i k}=-q_{k i}
$$

$p_{i k} \equiv x_{i} y_{k}-x_{k} y_{i}$, wobei auch $p_{i k}=-p_{k i}$,

sind bekanntlich die von Plücker zur Bestimmung der Geraden als Raumelement eingeführten homogenen Coordinaten derselben. Es bestehen zwischen ihnen die Identitäten

$$
\begin{aligned}
& q_{01} q_{23}+q_{20} q_{13}+q_{03} q_{12} \equiv 0 \\
& p_{01} p_{23}+p_{20} p_{13}+p_{03} p_{12} \equiv 0 .
\end{aligned}
$$

Zugleich lassen die Verbältnisse der $p$ und $q$ eine geometrische Deutung zu; man hat, wenn $\alpha, \beta, \gamma$ die Winkel sind, welche die Gerade mit den Coordinatenachsen macht:

und

$$
\cos \alpha: \cos \beta: \cos \gamma=q_{12}: q_{20}: q_{01}=p_{03}: p_{13}: p_{23}
$$

$$
n \cos \lambda: n \cos \mu: n \cos \nu=q_{133}: q_{13}: q_{23}=p_{12}: p_{20}: p_{01} \text {, }
$$

wobei $\lambda, \mu, \nu$ die Winkel bedeuten, welche eine durch die Gerade und den Coordinatenanfang gelegte Ebene mit den Coordinatenebenen oder eine auf ihr errichtete Senkrechte $s$ mit den Achsen macht, während $n$ die Länge der vom Coordinatenursprung auf die Gerade gefällten Normale ist. Zugleich sind dann auch die entsprechenden 
Glieder beider Reihen untereinander proportional und wird ihre $\mathrm{Zu}$ sammengehörigkeit, was die $p$ und $q$ anlangt, bestimmt durch die Gleichungen

und

$$
f \cdot p_{i k}=\frac{\partial Q}{\partial q_{i k}} \equiv q_{i^{\prime} k^{\prime}}
$$

$$
\boldsymbol{\varphi} \cdot q_{i k}=\frac{\partial \boldsymbol{P}}{\partial p_{i k}} \equiv p_{i^{\prime} k^{\prime}},
$$

worin $f$ und $\varphi$ Proportionalitätsfactoren, $P$ und $Q$ aber die linken Seiten der obigen Identitäten bezeichnen. Es können daher in jeder homogenen Gleichung zwischen $p$ dieselben mit Berücksichtigung dieser Beziehungen durch die $q$ ersetzt werden und umgekehrt.

Jede sechs Grössen, welche den obigen Identitäten genügen, dürfen $z u$ homogenen Coordinaten einer Geraden gemacht werden, die durch sie vollkommen bestimmt ist; sind dieselben von der Beschaffenheit, dass

$$
q_{12}^{2}+q_{20}^{2}+q_{01}^{2}=1
$$

ist, so stellen sie für diese Gerade wirklich $\cos \alpha, \ldots$ dar, und sollen dann ihre homogenen rechtwinkligen Coordinaten heissen. Es können solche aus den allgemeinen homogenen Coordinaten immer durch Division mit $\sqrt{q_{12}^{2}+q_{20}^{2}+q_{01}^{2}}$ hergestellt werden.

Von den allgemeinen Coordinaten $q$ werden $q_{12}=q_{20}=q_{01}=0$ für eine unendlich entfernte Gerade, während ihre übrigen drei Coordinaten in $\cos \lambda, \cos \mu, \cos \nu$ übergehen, wodurch dann die Ebene bestimmt ist, deren unendlich entfernten Theil die betreffende Gerade bildet; ist andrerseits $q_{03}=q_{13}=q_{23}=0$, so geht die zugehörige Gerade durch den Coordinatenanfang, und ist dann durch die drei übrigen Coordinaten, welche ihre Richtungscosinus ausdrücken, gegeben.

Diejenigen Geraden, deren Coordinaten einer Gleichung $n^{\text {ten }}$ Grades zwischen veränderlichen $p$ oder $q$ genügen, bilden nach Plücker einen Complex $n^{\text {ten }}$ Grades; diejenigen, welche zwei Complexen gemein sind, gehören zu einer Congruenz, und endlich die drei Complexen gemeinsamen erzeugen eine Regelfläche.

Von den vielen in der analytischen Geometrie vorkommenden Ausdrücken, in welchen die obigen Coordinaten der Geraden schon vorkommen oder durch Transformation sofort eingeführt werden können, und die also Complexe darstellen, ist einer der nächstliegenden die $\mathrm{Be}$ dingungsgleichung, unter welcher zwei Gerade, dargestellt durch die Gleichungen :

$$
\left\{\begin{array}{l}
u_{0} x+u_{1} y+u_{2} z+u_{3} p=0 \\
v_{0} x+v_{1} y+v_{2} z+v_{3} p=0
\end{array}\right\},
$$


sich schneiden, nämlich

$$
\left\{\begin{array}{l}
w_{0} x+w_{1} y+w_{2} z+w_{3} p=0 \\
r_{0} x+r_{1} y+r_{2} z+r_{3} p=0
\end{array}\right\}
$$

$$
\Sigma \pm u_{0} v_{1} w_{2} r_{3}=0
$$

Die Minoren zweiter Ordnung, in welche diese Determinante zerlegt werden kann, sind die Coordinaten jener beiden Geraden und es kann daher die vorige Bedingungsgleichung geschrieben werden:

$$
q_{03}^{\prime} q_{12}+q_{13}^{\prime} q_{20}+q_{23}^{\prime} q_{01}+q_{12}^{\prime} q_{03}+q_{20}^{\prime} q_{13}+q_{01}^{\prime} q_{23}=0 \text {, }
$$

wenn die Grössen $q$ die obige Bedeutung haben und analog

$$
q_{i k}^{\prime}=w_{i} r_{k}-w_{k} r_{i}
$$

gesetzt ist. Werden in dieser letzten Gleichung die Coordinaten $q$ der einen Geraden variabel angenommen, so stellt sie einen Complex ersten Grades dar und zwar den sogenannten speciellen linearen Complex, der also aus allen Geraden besteht, welche eine gegebene feste Gerade, die Axe des Complexes schneiden. Sind die als constant angenommenen Grössen $q^{\prime}$ die homogenen rechtwinkligen Coordinaten der Axe, so soll die Gleichung

$$
\begin{aligned}
& n_{1}\left\{\cos \lambda_{1} \cdot q_{12}+\cos \mu_{1} \cdot q_{20}+\cos \nu_{1} \cdot q_{01}\right\} \\
& +\cos \alpha_{1} \cdot q_{03}+\cos \beta_{1} \cdot q_{13}+\cos \gamma_{1} \cdot q_{23}=0
\end{aligned}
$$

die Gleichung des speciellen Complexes ersten Grades in der Normalform heissen und es kann daher die Gleichung desselben Complexes in der allgemeinen Form immer auf diese Normalform reducirt werden durch Multiplication mit

$$
\frac{1}{\sqrt{q_{12}^{\prime}+q_{20}^{\prime}+q_{01}^{\prime}}}
$$

Bleiben wir nun zunächst bei der Gleichung des speciellen Complexes erster Ordnung in der Normalform stehen, so wird die linke Seite derselben im Allgemeinen von Null verschieden sein, wenn darin die homogenen rechtwinkligen Coordinaten einer Geraden eingesetzt werden, und es liegt die Frage nahe, ob nicht für den Werth, welchen sie annimmt, eine geometrische Bedeutung gewonnen werden kann in ähnlicher Weise, wie das Substitutionsresultat der Coordinaten eines Punktes in die linke Seite einer in der Normalform gegebenen Gleichung einer Ebene die Entfernung des Punktes von der Ebene ausdrückt. Es ergiebt sich eine solche ganz einfach, wenn man den Grösen $q$ die ihnen als rechtwinkligen homogenen Coordinaten zukommenden Werthe giebt, wodurch die linke Seite der Complexgleichung übergeht in

$$
\begin{array}{r}
n_{1}\left\{\cos \alpha \cos \lambda_{1}+\cos \beta \cos \mu_{1}+\cos \gamma \cos \nu_{1}\right\} \\
+n\left\{\cos \alpha_{1} \cos \lambda+\cos \beta_{1} \cos \mu+\cos \gamma_{1} \cos \nu\right\}
\end{array}
$$


Die Coefficienten der $n$ stellen dann die Cosinus der Winkel dar, welche jede der gegebenen Geraden $g$ und $g$ mit einer auf der durch die anderen und den Coordinatenanfang gelegten Ebene errichteten Senkrechten $s_{1}$ resp. $s$ macht, sodass also für jene Substitutionsresultat geschrieben werden kann

$$
n_{1} \cos \left(g, s_{1}\right) \pm n \cos \left(g_{1}, s\right),
$$

wo, wie auch im Folgenden, das obere oder untere Vorzeichen zu nehmen ist, je nachdem beide Gerade auf verschiedenen oder auf derselben Seite des Coordinatenanfangs liegen. Man erhält jedoch noch eine andere vom Coordinatensystem unabhängige Interpretation, aus der Lösung der Aufgabe die kürzeste Entfernung $N$ zweier Geraden $g_{0}$ und $g_{1}$ durch ihre rechtwinkligen homogenen Coordinaten auszudrücken. Wenn die Gleichungen der beiden Geraden in der Form

vorausgesetzt werden, so ist

$$
\frac{x-\xi}{\cos \alpha}=\frac{y-\eta}{\cos \beta}=\frac{z-\xi}{\cos \gamma}
$$

$$
\begin{aligned}
N & =\left\{\left(\xi_{0} \pm \xi_{1}\right)\left(\cos \beta_{0} \cos \gamma_{1}-\cos \beta_{1} \cos \gamma_{0}\right)\right. \\
& +\left(\eta_{0} \pm \eta_{1}\right)\left(\cos \gamma_{0} \cos \alpha_{1}-\cos \gamma_{1} \cos \alpha_{0}\right) \\
& \left.+\left(\xi_{0} \pm \xi_{1}\right)\left(\cos \alpha_{0} \cos \beta_{1}-\cos \alpha_{1} \cos \beta_{0}\right)\right\}: \sin \theta_{1}
\end{aligned}
$$

wo $\Theta$ den Neigungswinkel beider Geraden bedeutet. Führt man nun für die Coordinaten $\xi_{0}, \eta_{0}, \xi_{0}$ und $\xi_{1}, \eta_{1}, \xi_{1}$ der beiden auf jenen Geraden beliebig zu wählenden Punkte die der Fusspunkte der vom Coordinatenanfang auf sie gefällten Perpendikel ein, setzt also

$$
\xi=n \cos \varrho, \eta=n \cos \sigma, \xi=n \cos \tau,
$$

wo $\varrho, \sigma, \tau$ die Winkel sind, welche diese Perpendikel mit den Axen machen, so geht der vorige Ausdruck für $N$ über in $\left[n_{1}\left\{\alpha_{0}\left(\sigma_{1} \gamma_{1}-\tau_{1} \beta_{1}\right)+\beta_{0}\left(\tau_{1} \alpha_{1}-\varrho_{1} \gamma_{1}\right)+\gamma_{0}\left(\varrho_{1} \beta_{1}-\sigma_{1} \alpha_{1}\right)\right\}\right.$

$$
\left. \pm n_{0}\left\{\alpha_{1}\left(\sigma_{0} \gamma_{0}-\tau_{0} \beta_{0}\right)+\beta_{1}\left(\tau_{0} \alpha_{0}-\varrho_{0} \gamma_{0}\right)+\gamma_{1}\left(\varrho_{0} \beta_{0}-\sigma_{0} \alpha_{0}\right)\right\}\right]: \sin \Theta
$$

mit Weglassung der cos-Zeichen. Da num die Senkrechten $n$ in den durch die Geraden und den Anfangspunkt gehenden Ebenen liegen, also

$$
\cos \lambda: \cos \mu: \cos \nu
$$

$=\cos \sigma \cos \gamma-\cos \tau \cos \beta: \cos \tau \cos \alpha-\cos \rho \cos \gamma: \cos \rho \cos \beta-\cos \sigma \cos \alpha$ ist, ausserdem

$$
\begin{gathered}
(\sigma \gamma-\tau \beta)^{2}+(\tau \alpha-\rho \gamma)^{2}+(\rho \beta-\sigma \alpha)^{2} \\
\equiv\left(\rho^{2}+\sigma^{2}+\tau^{2}\right)\left(\alpha^{2}+\beta^{2}+\gamma^{2}\right)-(\rho \alpha+\sigma \beta+\tau \gamma) \equiv 1
\end{gathered}
$$

ist, so stellen jene Ausdrücke $\cos \sigma \cos \gamma-\cos \tau \cos \beta, \ldots$ wirklich $\cos \lambda, \ldots$ dar und man erhält also

\section{$N \sin \Theta$}

$$
\begin{array}{r}
=n_{1}\left(\cos \alpha_{0} \cos \lambda_{1}+\cos \beta_{0} \cos \mu_{1}+\cos \gamma_{0} \cos \nu_{1}\right) \\
\pm n_{0}\left(\cos \alpha_{1} \cos \lambda_{0}+\cos \beta_{1} \cos \mu_{0}+\cos \gamma_{1} \cos \nu_{0}\right)
\end{array}
$$


eine Gleichung, deren rechte Seite mit der linken Seite der Gleichung eines speciellen Complexes in der Normalform übsreinstimmt, in welche rechtwinklige homogene Coordinaten einer Geraden substituirt sind. Hiermit ist denn Folgendes bewiesen:

Werden in die linke Seite der Gleichung eines speciellen Complexes erster Ordnung in der Normalform die rechtwinkligen homogenen Coordinaten einer beliebigen Geraden eingesetzt, so stellt sie das Product aus der Entfernung jener Geraden von der Axe des Complexes in den Sinus ihres Neigungswinkels gegen die Axe dar*). der Satz:

Durch Vergleichung dieser Deutung mit der vorhergehenden folgt

Fällt man von einem beliebigen Punkte Perpendikel auf zwei gegebene Gerade, so ist die Summe der Producte aus der Länge eines jeden derselben in den Cosinus des Winkels, welchen eine zu ihm und der Geraden, worauf es gefällt ist, construirte Normale mit der jedesmaligen andern macht, constant, und gleich dem Product aus der Entfernung beider Geraden in den Sinus ihres Neigungswinkels.

Es lässt sich derselbe auch leicht mittelst trigonometrischer Betrachtungen darthun; auch ist er in besonderen Fällen, z. B. wenn beide Gerade parallel sind, ohne Weiteres evident. Ein für uns später wichtiger Specialfall ergiebt sich, wenn der angenommene Punkt auf einer der beiden Geraden selbst liegt, nämlich:

Durchläuft ein Punkt eine feste Gerade, so ist das Product aus seiner Entfernung von einer andern festen Geraden in den Sinus des Winkels, welchen die erstere mit einer durch den Punkt und die zweite Gerade gelegten Ebene macht, constant, oder anders ausgedrückt:

Wird eine Gerade parallel zu sich selbst so verschoben, dass ihre Entfernung von einer festen Geraden unverändert bleibt, so ist für einen beliebig auf der letzteren gewählten Punkt das Product aus dem von ihm auf die sich bewegende Gerade gefüllten Perpendikel in den Cosinus des Winkels, den eine zu diesem Perpendikel und der sich bewegenden Geraden gedachte Normale mit der festen Geraden macht, constant.

*) Dieser Satz bringt eine gewisse Gruppirung in die zu dem speciellen Complex erster Ordnung gehörigen Geraden; denn soll

$$
N \sin \theta=0
$$

sein, so muss entweder $\sin \theta=0$ sein, d. h. die Gerade parallel zur Axe des Complexes, oder $n=0, \mathrm{~d} . \mathrm{h}$. die Axe von ihr in endlicher Entfernung geschnitten werden. Im letateren Falle ordnen sich dann die Geraden, für welche sin $\theta$ denselben constanten Werth hat, in leicht zu übersehender Weise an. 
Dadurch, dass man die bei diesem letzteren Ausdrucksweise zu denkende Raumfigur um die feste Gerade rotiren lässt, sowie endlich in dem constanten Werthe $N \sin \theta$ beide Factoren variabel denkt, erhält man noch folgende zwei Erweiterungen des Satzes:

Wird eine Gerade um eine feste Axe so gedreht und längs ihr verschoben, dass ihre Entfernung und Neigung gegen sie dieselben bleiben, so hat das Product des von einem Punkte jener Axe auf die Gerade gefällten Perpendikels in den Cosinus des Winkels, welchen die Axe mit einer auf der durch jenen Punkt und die Gerade gelegten Ebene errichteten Normalen macht, immer denselben Werth;

endlich:

Bei allen Geraden, für die das Product aus ihrer Entfernung von einer festen Geraden in den Sinus ihres Neigungswinkels gegen dieselbe constant ist, ist auch das Product aus der von einem beliebigen Punkte der festen Geraden auf eine von ihnen gefällten Senkrechten in den Cosinus des Winkels, den die feste Gerade mit einer auf der durch jenen Punkt und die andere Gerade gelegten Ebene errichteten Normalen macht, constant.

Die zuletzt gefundené Bedeutung der linken Seite der speciellen Complexgleichung ermöglicht die Aufstellung eines tetraedrischen Coordinatensystems für die Raumgerade. Geht man nämlich aus von den Gleichungen :

$$
\left\{\begin{array}{l}
u_{0} x+u_{1} y+u_{2} z+u_{3} p=0 \\
v_{0} x+v_{1} y+v_{2} z+v_{3} p=0
\end{array}\right\}
$$

derselben in dem bisher benutzten rechtwinkligen System, so dienen zu ihrer Uebertragung in ein tetraedrisches Punktcoordinatensystem die Formeln:

$$
\begin{aligned}
& x=x_{0} X+x_{1} Y+x_{2} Z+x_{3} P \\
& y=y_{0} X+y_{1} Y+y_{2} Z+y_{3} P \\
& z=z_{0} X+z_{1} Y+z_{2} Z+z_{3} P \\
& p=p_{0} X+p_{1} Y+p_{2} Z+p_{3} P
\end{aligned}
$$

worin die Constanten $\frac{x_{0}}{p_{0}}, \frac{y_{0}}{p_{0}}, \cdots$ die Goordinaten der Eckpunkte des Fundamentaltetraeders sind. Durch Substitution dieser Werthe für $x, y, z, p$ in die Gleichuugen unserer Geraden gehen diese über in

$$
\left\{\begin{array}{l}
U_{0} X+U_{1} Y+U_{2} Z+U_{3} P=0 \\
V_{0} X+V_{1} Y+V_{2} Z+V_{3} P=0
\end{array}\right\}
$$

wenn die Coefficienten $U$ und $V$ folgende Bedeutung haben:

$$
\begin{aligned}
& U_{n}=x_{n} u_{0}+y_{n} u_{1}+z_{n} u_{2}+p_{n} u_{3}, \\
& V_{n}=x_{n} v_{0}+y_{n} v_{1}+z_{n}^{\prime} v_{2}+p_{n} v_{3} .
\end{aligned}
$$

Werden nun aus ihnen die Coordinaten $Q$ unserer Geraden für das 
neue System gebildet, so ergiebt sich folgender Zusammenhang derselben mit den frühern $q$, es ist

$Q_{i k}=\left(x_{i} y_{k}\right) q_{01}+\left(y_{i} z_{k}\right) q_{12}+\left(z_{i} x_{k}\right) q_{02}+\left(x_{i} p_{k}\right) q_{03}+\left(y_{i} p_{k}\right) q_{13}+\left(z_{3} p_{k}\right) q_{23}$, wo $\left(x_{i} y_{k}\right)=x_{i} y_{k}-\dot{x}_{k} y_{i}, \cdots$ ist und also die Coefficienten der $q$ die Coordinaten je einer Kante des Fundamentaltetraeders im ursprünglichen System sind. Durch passende Wahl desselben wird man es einrichten können, dass diese Ausdrücke $\left(x_{i} y_{k}\right) \ldots$ für sämmtliche Kanten die bomogenen rechtwinkligen Coordinaten in dem oben festgesetzten Sinne sind, sodass, wenn dann auch die $q$ als solche angenommen werden, die Ausdrücke für die $Q$ mit den linken Seiten von Gleichungen specieller linearer Complexe in der Normalform übereinstimmen, in welche die rechtwinkligen Coordinaten einer Geraden eingesetzt sind, und also die folgende Definition der $Q$ aufgestellt werden kann:

Die tetraedrischen Coordinaten einer Geraden sind die Producte aus den Entfernungen derselbeu von den Kanten des Fundamentaltetraeders in die Sinusse ihrer betreffenden Neigungswinkel gegen diese Kanten.

Da für sie auch wieder die identische Relation

$$
Q_{01} Q_{23}+Q_{02} Q_{31}+Q_{03} Q_{12}=0
$$

besteht, so ergeben sich daraus Beziehungen zwischen einer Geraden resp. Punkten einer Geraden von den Kanten eines Tetraeders, auf welche wir hier nicht weiter eingehen. Eine Verallgemeinerung der 'Tetraedercoordinaten, wie wir sie eben definirt haben, sind tetraedrische Verhältnisscoordinaten, von denen man dann leicht zu den Plücker'schen Verhältnisscoordinaten gelangen kann, indem man, wie um von den tetraedrischen Punktcoordinaten auf Cartesische zu kommen, eine Seitenebene des Fundamentaltetraeders, die wir mit 012 bezeichnen wollen, in unendliche Ferne rücken lässt, während die 3 anderen zu einander senkrecht sind. Denn es können zunächst für die Verhältnisse der auf die drei Schnittlinien der letzteren, d. h. die Axen $O X, O Y, O Z$ bezüglichen Producte von der Form $N \sin \Theta$ nach einem der obigen Sätze Ausdrücke substituirt werden, welche proportional sind zu $n \cos \lambda, n \cos \mu, n \cos \nu$, wenn $n, \lambda, \mu, \nu$ die frühere Bedeutung haben. Für die in unendliche Ferne entrückten Kanten aber ist

$$
\left(x_{\theta} p_{1}\right)=\left(y_{0} p_{1}\right)=\left(z_{0} p_{1}\right)=\left(x_{1} p_{2}\right)=\left(y_{1} p_{2}\right)=\left(z_{1} p_{2}\right)=\left(x_{2} p_{0}\right)=\left(y_{2} p_{0}\right)=\left(z_{2} p_{0}\right)=0
$$

und also

$$
Q_{i k}=\left(x_{i} y_{k}\right) q_{01}+\left(y_{i} z_{k}\right) q_{12}+\left(z_{i} x_{k}\right) q_{02},
$$

$$
[i k=01,12,20] \text {, }
$$

welche Ausdrücke den Cosinus der Winkel proportional sind, die unsere Gerade mit auf den Ebenen 301, 312, 320 errichteten Normalen, 
d. h. den Axen $O Z, O X, O Y$ macht; es ist deshalb

$$
Q_{01}: Q_{22}: Q_{20}=\cos \gamma: \cos \alpha: \cos \beta \text {. }
$$

Wenden wir uns nun zur Untersuchung des allgemeinen linearen Complexes, so wird ein solcher repräsentirt durch die Gleichung:

$$
A q_{12}+B q_{20}+C q_{01}+D q_{03}+E q_{13}+F q_{23}=0
$$

wo $A, B \ldots$ beliebige Constante sind; er geht in den speciellen über, wenn zwisehen ihnen die Relation

$$
A D+B E+C F \equiv 0
$$

stattfindet, d. h. jene Coefficienten Coordinaten einer Geraden, der Axe des speciellen Complexes sind. Die Gleichung des Complexes soll in der Normalform gegeben heissen, wenn

$$
D^{2}+E^{2}+F^{2}=1
$$

ist; daun können die Grössen $D, E, F$ aufgefasst werden als Cosinus der Winkel, welche eine Gerade mit den Axen macht, während $A, B, C$ angesehen werden dürfen als Producte aus der Entfernung einer andern Geraden rom Coordinatenanfang in die Cosinus der Winkel, welche eine auf der durch sie und den Anfangspunkt gelegten Ebene errichtete Normale mit den Axen einschliesst. Zur Reduction der allgemeinen Complexgleichung auf die Normalform dient der Factor

$$
\frac{1}{\sqrt{\bar{D}^{2}+\bar{E}^{2}+\bar{F}^{\overline{2}}}} \text {. }
$$

Um nun zunächst für die einem allgemeinen Conplex angehörigen Geraden ein Gesetz zu finden, entsprechend der Bestimmung des speciellen Complexes durch die Gleichung $N$ sin $\Theta=0$, braucht man nur für zwei durch ihre Coordinaten $q^{\prime}$ und $q^{\prime \prime}$ bestimmte feste Gerade weitere Gerade zu suchen, welche der Bestimmung

$$
N_{1} \sin \theta_{1}=k N_{11} \sin \theta_{11}
$$

wo $N$ und $\Theta$ die frïhere Bedeutung in Bezug anf die beiden festen Geraden haben und $k$ eine Constante ist, genügen, denn diese Bedingungsgleichung in Furiction der Coordinaten jener drei Geraden ausgedrückt, giebt

$$
\begin{aligned}
\left(q_{23}^{\prime}-k q_{23}^{\prime \prime}\right) q_{01} & +\left(q_{03}^{\prime}-k q_{03}^{\prime \prime}\right) q_{12}+\left(q_{13}^{\prime}-k q_{13}^{\prime \prime}\right) q_{20}+\left(q_{12}^{\prime}-k q_{12}^{\prime \prime}\right) q_{03} \\
& +\left(q_{20}^{\prime}-q_{20}^{\prime \prime}\right) q_{13}+\left(q_{01}^{\prime}-k q_{01}^{\prime \prime}\right) q_{23}=0
\end{aligned}
$$

eine Gleichung, die einen allgemeinen linearen Complex darstellt, da zwischen den Coefficienten der $q$ die bewusste Identität $\left(q_{03}^{\prime}-k q_{03}^{\prime \prime}\right)\left(q_{12}^{\prime}-k q_{12}^{\prime \prime}\right)+\left(q_{13}^{\prime}-k q_{13}^{\prime \prime}\right)\left(q_{20}^{\prime}-k q_{20}^{\prime \prime}\right)+\left(q_{23}^{\prime}-k q_{23}^{\prime \prime}\right)\left(q_{01}^{\prime}-k q_{01}^{\prime \prime}\right)=0$ im Allgemeinen nicht stattfindet. Hieraus folgt:

Ein allgemeiner linearer Complex besteht aus allen Geraden, für welche die Producte aus ihren Entfernungen von zwei festen Geraden in die Sinusse ihrer betreffenden Neigungswinkel gegen dieselben ein constantes Verhältniss haben. 
Bei derartiger Bestimmung des Complexes mag dieses constante Verhältniss der Modul, die beiden gegebenen Geraden die Directricen des Complexes heissen; je nach dem Werthe des Moduls $k$ erhält man bei denselben Directricen eine ganze Reihe von Complexen, welchen dieselbe Congruenz, bestehend aus allen die Directricen schneidenden. Geraden, gemein ist, und die nach Plücker's Benennung eine zwejgliederige Gruppe bilden. Hierunter befinden sich zwei specielle mit den Directricen als Axen für die Werthe $k=0$ und $k=\infty$.

Es lassen sich leicht für jeden gegebenen Complex Gerade von der angegebenen Bedeutung für ihn ermitteln; denn vergleicht man die Coefficienten seiner Gleichung mit den obigen $q^{\prime}-k q^{\prime \prime}$, so müssen die $q^{\prime}$ und $q^{\prime \prime}$ zunächst den Gleichungen

$$
\begin{aligned}
& q_{03}^{\prime}-k q_{03}^{\prime \prime}=A, q_{13}^{\prime}-k q_{13}^{\prime \prime}=B, q_{23}^{\prime}-k q_{23}^{\prime \prime}=C, \\
& q_{12}^{\prime}-k q_{12}^{\prime \prime}=D, q_{20}^{\prime}-k q_{20}^{\prime \prime}=E, q_{01}^{\prime}-k q_{01}^{\prime \prime}=F,
\end{aligned}
$$

und ausserdem den bekannten Identitäten genügen. Man findet dann, dass, wenn etwa die $q^{\prime \prime}$ also die eine Gerade beliebig gewählt werden, sich für $k$ der einzige Werth

$$
k=-\frac{A D+B E+C F}{A q_{12}^{\prime \prime}+B q_{20}^{\prime \prime}+C q_{01}^{\prime \prime}+D q_{03}^{\prime \prime}+\bar{E} q_{13}^{\prime \prime}+F q_{23}^{\prime \prime}}
$$

ergiebt, folglich die $q^{\prime}$ und somit die andere Gerade eindeutig bestimmt sind. Also:

Durch eine als Directrix eines gegebenen Complexes beliebig gewählte Gerade ist die andere Directrix und der zugehörige Modul bestimmt.

Bei wirklicher Bildung der $q^{\prime}$ ergiebt sich, dass jedem Paar Gerade, welche Directricen eines Complexes sind, zugleich die Eigenschaft zukommt, weshalb sie von Plücker conjugirte Polarên in Bezug auf den Complex genannt werden.

Eine der augenfälligsten Eigenschaften des speciellen linearen Complexes ist die, dass, wenn man den ganzen Complex längs seiner Axe verschiebt und um dieselbe dreht, er unverändert bleibt, indem alle ihm angehörenden Geraden nach der Verschiebung und Drehung mit anderen Geraden des Complexes zur Deckung kommen, welche vorher ihre Stelle einnahmen. Die Gleichung des Complexes kann sich bei einer diesem Vorgange entsprechenden Coordinatenänderung nur um einen constanten Factor ändern. Um nun zu untersuchen, ob auch bei dem allgemeinen Complex eine derartige Axe existirt, kehren wir zurück zu den Gleichungen, welche die Abhängigkeit der Constanten des Complexes von Directricen und Modul vermitteln. Sollen zwei beliebige Gerade Directricen eines bestimmten Complexes sein, so muss

$$
q_{12}^{\prime}-k q_{12}^{\prime \prime}=\varrho D, q_{20}^{\prime}-k q_{20}^{\prime \prime}=\varrho E, q_{01}^{\prime}-k q_{01}^{\prime \prime}=\varrho F
$$


sein, aus welchen Gleichungen durch Elimination des Proportionalitätsfactors $\varrho$ und des für verschiedene Directricen wechselnden Moduls $k$ folgt:

$$
\left(q_{01}^{\prime} q_{20}^{\prime \prime}-q_{20}^{\prime} q_{01}^{\prime \prime}\right) D+\left(q_{12}^{\prime} q_{01}^{\prime \prime}-q_{01}^{\prime} q_{12}^{\prime \prime}\right) E+\left(q_{20}^{\prime} q_{12}^{\prime \prime}-q_{12}^{\prime} q_{20}^{\prime \prime}\right) F=0 \text {, }
$$

d. h. als Satz:

Die Linie kürzester Entfernung von irgend zwei zusammengehörigen Directricen eines Complexes 1. Ordnung ist einer bestimmten Ebene parallel, oder was dasselbe sagt, zu einer bestimmten Richtung normal, deren Richtungscosinus bestimmt sind durch $D: E: F$. Hieraus folgt nun für die Möglichkeit der Axe überhaupt, dass sie jener Richtung parallel sein muss, indem nur dann bei einer Verschiebung längs ihr resp. Drehung um sie auch, wie es sein muss, alle Directricenpaare des Complexes mit andern zur Deckung kommen können. Sie muss ferner alle jene Linien kürzester Entfernung schneiden. Wird nun die Gleichung des Complexes in der Normalform, und werden die Coordlnaten einer zur Axenrichtung parallelen Directrix als rechtwinklige homogene vorausgesetzt, d. h. $q_{12}^{\prime}=D, q_{20}^{\prime}=E, q_{01}^{\prime}=F$, so folgt unmittelbar für die zugehörige Directrix $q_{12}^{\prime \prime}=q_{20}^{\prime \prime}=q_{01}^{\prime \prime}=0$. Dies giebt den Satz:

Jede zur Axe parallele Gerade hat ihre conjugirte Directrix im Unendlichen.

Der zugehörige Werth des Moduls ist:

$$
k=-\frac{A D+B E+C F}{D q_{03}^{\prime \prime}+\bar{E} q_{13}^{\prime \prime}+F q_{23}^{\prime \prime}}
$$

und wird erst dann bestimmt sein, wenn durch bestimmte Werthe von

$$
q_{03}^{\prime}, q_{13}^{\prime}, q_{23}^{\prime}
$$

auch die entsprechenden Coordinaten der zugehörigen Directrix fixirt oder umgekehrt durch beliebige Wahl der letzteren die ersteren bedingt sind. Da, wie oben gezeigt, zu jeder Geraden in Bezug auf den Complex nur eine conjugirte gehört, so muss die zur Axe gehörige Directrix bei jeder Verschiebung und Drehung des Complexes längs resp. um die Axe immer dieselbe bleiben, welcher Bedingung sich offenbar nur dadurch genügen lässt, dass sie senkrecht zur Axenrichtung in unendlicher Ferne angenommen wird. Diese Annahme liefert die zur Bestimmung der noch unbekannten drei Coordinaten der Axe nöthigen Daten; die Axe bildet dann mit ihrer unendlich entfernten conjugirten Normalen ein Directricenpaar von der Beschaffenheit, dass der Complex bei jeder Verschiebung und Drehung längs resp. um eine von ihnen unverändert bleibt.

Setzen wir nun, um die drei noch unbekannten Coordinaten der Axe zu ermitteln, wie es, wenn die zugehörige Directrix zu ihr normal sein soll, sein muss, $q_{03}^{\prime \prime}=D, q_{13}^{\prime \prime}=E$, $q_{23}^{\prime \prime}=\dot{F}$, so wird 


$$
k=-\frac{A D+B E+C F^{\prime}}{D^{2}+E^{2}+F^{2}}
$$

und ergeben sich für diesen besondern Werth des Moduls, den Plü cker mit $k$ bezeichnet und den Parameter des Complexes genannt hat

$$
\begin{aligned}
& q_{03}^{\prime}=(A E-B D) E-(C D-A F) F \\
& q_{13}^{\prime}=(B F-C E) F-(A E-B D) D \\
& q_{23}^{\prime}=(C D-A F) D-(B F-C E) E
\end{aligned}
$$

als die gesuchten drei Coordinaten der Axe, und daraus ihre Entfernung $n$ rom Coordinatenanfang

$$
=\sqrt{q_{03}^{\prime 2}+q_{13}^{\prime 2}+q_{23}^{\prime 2}}
$$

Ist die Gleichung des Complexes in der Normalform gegeben, wie wir eben vorausgesetzt, so ist der Werth des Parameters $A D+B E+C F$. Soll die Axe des Complexes durch den Coordinatenanfang gehen, so müssen ihre drei vorher bestimmten Coordinaten Null werden, und findet sich dann durch Vergleichung der sie liefernden Ausdrücke:

$$
A-k D, B-k E, C-k F
$$

mit Null die in diesem Falle zwischen den Constanten des Complexes stattfindende Relation

$$
A: B: C=D: E: F \text {. }
$$

Dieses Resultat ergiebt sich auch durch rein geometrische Betrachtungen, wenn man für die linke Seite der Complexgleichung schreibt

$$
\begin{aligned}
& n(\cos a \cos \lambda+\cos b \cos \mu+\cos c \cos \nu) \\
+ & P(\cos L \cos \alpha+\cos M \cos \beta+\cos N \cos \gamma),
\end{aligned}
$$

wo $\cos a=A, \ldots, P \cos L=D, \ldots$ gesetzt sind, dann voraussetzt, sie werde für eine bestimmte Gerade $\equiv 0$, und endlich von den obigen Sätzen über die von Punkten einer Geraden auf eine andere gefällten Senkrechten Gebrauch macht. Dabei ergeben sich noch weitere Resultate, wie z. B. $n \operatorname{tg} \lambda=k$, wo $n$ die Entfernung einer Geraden des Complexes von der Achse, $\lambda$ den Winkèl zwischen beiden und $k$ den Parameter bezeichnet.

Um die Lage der Achse eines Complexes bei gegebenen Directricen 0 und 1 , sowie gegebenem Modul $k$ festzustellen, nehmen wir die rechtwinkligen Coordinaten jener beiden Geraden als gegeben an, dann ist

$D: E: F=\cos \alpha_{0}-k \cos \alpha_{1}: \cos \beta_{0}-k \cos \beta_{1}: \cos \gamma_{0}-k \cos \gamma_{1}$,

d. h. in Worten:

Die Axe des Complexes theilt den Winkel zwischen den Directricen so, dass das Verhältniss der ${ }^{*}$ Sinus der Theilwinkel dem Modul gleich ist.

Wir wissen ferner, dass sie die kürzeste Verbindungslinie jener beiden 
Geraden schneidet und dass, wenn sie durch den Anfangspunkt geht, zwischen den Constanten des Complexes die Gleichung

$$
A: B: C=D: E: F
$$

besteht; es kann daher das Verhältniss, in welchem die linie kürzester Entfernung von der Axe getheilt wird, am einfachsten gefunden werden, indem man einen Complex componirt für einen gegebenen Modul $k$, mit Zugrundelegung eines Coordinatensystems, dessen $Y$-Axe die Linie kürzester Entfernung der als Directricen gegebenen Geraden 0 und 1 ist, und dessen $X$-Axe durch einen noch zu bestimmenden Punkt auf ihr parallel zu einer jener Geraden, etwa zu 0) geht, während die $Z$-Axe zu beiden senkrecht ist. Die Gerade 0 hat dann die rechtwinkligen Coordinaten $1,0,0,0,0, n$ und es sind $\cos \alpha_{1}, 0, \sin \alpha_{1}, n_{1} \sin \alpha_{1}, 0, n_{1} \cos \alpha_{1}$ die von 1 , sodass durch $\alpha_{1}$ auch zugleich der Neigungswinkel beider Geraden ausgedrückt ist. Als Constanten des Complexes ergeben sich dann:

$$
\begin{aligned}
& A=-k n_{1} \sin \alpha_{1}, B=0, C=n-k n_{1} \cos \alpha_{1}, \\
& D=1-k \cos \alpha_{1}, E=0, F=-k \sin \alpha_{1},
\end{aligned}
$$

und aus der Gleichung $A: C=D: F$ folgt:

$$
\frac{n}{n_{1}}=\frac{k(\cos \alpha-k \cos 2 \alpha)}{1-k \cos \alpha},
$$

sodass nun die Lage der Axe allein abhängt von der Entfernung und Neigung der Directricen und rom Modul.

Schliesstich sei bemerkt, dass aus den gefundenen Resultaten sich sehr einfach eine Reihe von Sätzen über die zweigliederige Complexgruppe ergiebt, die sich in Plücker's Raumgeometrie pag. $62 \mathrm{ff}$. finden. 